\title{
miR-7111-5p and miR6870-5p may be Potential Biomarkers for Oral Squamous Cell Carcinoma
}

\author{
(1) Mona SARAEI,' (1) Ata GARAJEI, ${ }^{2,3}$ (I) Faranak JAMSHIDIAN' \\ 'Department of Biology, Islamic Azad University East Tehran Branch (Ghiamdasht), Faculty of Basic Sciences, Tehran-Iran \\ ${ }^{2}$ Department of Oral and Maxillofacial Surgery, Tehran University of Medical Sciences Faculty of Dentistry, Tehran-Iran \\ ${ }^{3}$ Department of Head and Neck Surgical Oncology and Reconstructive Surgery, The Cancer Institute, Tehran University of Medical \\ Sciences Faculty of Medicine,Tehran-Iran
}

\begin{abstract}
OBJECTIVE
MicroRNAs (miRs) are small, non-coding RNAs that are involved in tumor development and progression in multiple cancers with the action as oncomiRs or tumor suppressor miRs. Therefore, miRNAs have become potential biomarkers in the diagnosis and treatment of cancer. The previous studies have revealed that miR7111-5p and miR6870-5p are abnormally expressed in various types of cancer but in oral squamous cell carcinoma (OSCC) has not been elucidated. The main objective of our study was to evaluate the expression alternations of miR7111-5p and miR6870-5p as well as serum response factor (SRF) in OSCC tissues compared with adjacent normal oral epithelial tissues.
\end{abstract}

\section{METHODS}

SRF was identified as a potential target gene of miR7111-5p and miR6870-5p using the TargetScan, Mirwalk, and Mirdb miRNA-target prediction tools. SRF, miR7111-5p, and miR6870-5p expression levels were examined by RT-qPCR in OSCC compared with normal tissues.

\section{RESULTS}

Our results showed that miR7111-5p and miR6870-5p were significantly downregulated in 30 pairs of OSCC tissue samples $(\mathrm{p}<0.05)$ relative to adjacent normal oral tissues. In the present study, we found that the high expression level of $S R F$ was negatively correlated with the down regulation of miR7111-5p and miR6870-5p and was statistically correlated with the tumor stage.

\section{CONCLUSION}

miR7111-5p, miR6870-5p, and SRF may be potential prognostic biomarkers for OSCC diagnosis and monitoring.

Keywords: miR6870-5p; miR7111-5p; oral squamous cell carcinoma; serum response factor gene. Copyright $\odot$ 2021, Turkish Society for Radiation Oncology

\section{Introduction}

Oral squamous cell carcinoma (OSCC) is a global public health issue; it is the eighth most common type of oral cavity malignancy for men and the fifth among women worldwide. [1] Delay in the diagnosis and the relatively high tumor recurrence rates cause a 5-year survival rate of $<50 \%$ among OSCC patients.[2] The disease is the sixth most common cancer in the world, with more than 300.000 new cases reported annually. To date, several studies have reported a strong connection between environmental carcinogens, such as smoking, alcohol consumption, and the use of betel quid with increases the risk of OSCC.[3] 
Considering the asymptomatic nature of oral cancer at its early stage, early detection of OSCC plays a critical role in prognosis and reduces treatment-related morbidity and mortality. [4] Recent attention has focused on the potential application of biomarkers for patient assessment in multiple clinical stages. Cancer biomarkers can be used to estimate the risk of cancer, screen for occult primary cancers, distinguish benign from malignant tumors, diagnose cancer, develop a prognosis, detect recurrence, and assess response to therapy.[5]

MicroRNAs (miRNAs) have a molecular regulatory role and have emerged as diagnostic biomarkers of various human cancers, including OSCC. Several studies have recently investigated the association between the expression levels of some of these miRNAs and the initiation, progression, survival rate, and metastatic potential of cancer.[6,7] miRNAs are small non-coding RNAs (18-25 nu-cleotides long) that negatively regulate gene function at the posttranscriptional level of target genes by suppressing translation or degradation of the target mRNA. $[6,8]$ A number of studies have identified that increased expression of miRs in OSCC and OSCC-derived cells compared to normal tissues have known as the most prevalent risk factors in the OSCC development and tumorigenesis. [9-13]

Several miRs are characterized to act as tumor suppressors and/or oncogenes in OSCC. They regulate gene expression through interaction with components in the signaling pathways influencing cell proliferation, differentiation, migration, apoptosis, and other important involved mechanisms at cancer development.

Identification of miRNA expression patterns in OSCC compared to normal tissues can lead to earlystage diagnosis and prognosis.[14]

Serum response factor $(S R F)$ is a MAD-box containing transcription factor which has an important role in the regulation of many involved-specific genes in cell growth, proliferation, differentiation, and apoptosis.[15] It has been firmly established that $S R F$ is a direct target for regulation by miRNAs and also executes its regulatory role in microRNA expression and biogenesis where miRNAs are direct transcriptional targets of $S R F .[16,17]$ A previous study has reported that miR7111-5p and miR6870-5p were upregulated in OSCC[8] but the role of two miRs in the regulation of $S R F$ expression in OSCC remains elusive.

This study was designed to investigate the expression of miR7111-5p, miR6870-5p, and SRF as their target gene as well as to identify their possible expression association in OSCC and normal tissue.

\section{Materials and Methods}

\section{Patients and Tissue Samples}

In this study, a total of 30 pairs of frozen OSCC tissues and adjacent normal oral epithelial tissues were used. Informed consent was obtained from all patients for the use of clinical specimens and the present study was approved by the Research Medical Ethics Committee of Imam Khomeini Hospital. Patients' samples were collected from patients with OSCC by the Iran National Tumor Bank, Cancer Institute, Imam Khomeini Hospital, Tehran. The clinico-pathological characteristics of patients are shown in Table 1. Matched adjacent normal tissues were obtained from the OSCC patients after surgery and immediately frozen in liquid nitrogen and stored at $-80^{\circ} \mathrm{C}$ until RNA extraction.

\section{Predicting microRNA Target Genes}

The TargetScan (http://www.targetscan.org), Mirwalk (http://mirwalk.uni-hd.de), and miRDB (http://mirdb. org) databases were used to predict potential miRNA target genes. The selected gene for both miRNAs was subjected to analysis.

\section{RNA Extraction and Quantitative RT-PCR (qRT- PCR)}

The total RNA was extracted from frozen tissue samples using RiboEx ${ }^{\mathrm{Tm}}$ reagent (GeneAll Biotechnology, Korea), according to the manufacturer's instructions. In addition, the extracted RNA was treated with RNAse-free DNAase to eliminate any genomic DNA contamination. The quality and quantity of extracted RNAs were evaluated using 1.5\% agarose gel electrophoresis and Nanodrop (Thermo scientificNanodrop 2000) spectrophotometer analyses, respectively. miRNA was reverse transcribed with $1 \mu \mathrm{g}$ total RNA using BioFACT cDNA Synthesis kit (Daejeon, South Korea), according to the manufacturer's protocol. PCR was performed on an Exicycler ${ }^{\text {Tw }} 96$ thermocycler (Bioneer, Korea) using a SYBR-Green kit (Ampliqon). Quantitative real-time PCR on miRNAs and SRF was conducted with the following thermal cycling profile at $95^{\circ} \mathrm{C}$ for $2 \mathrm{~min}$, followed by 40 cycles comprising $95^{\circ} \mathrm{C}$ for $15 \mathrm{~s}, 60^{\circ} \mathrm{C}$ for $30 \mathrm{~s}$, and $72^{\circ} \mathrm{C}$ for $20 \mathrm{~s}$. U6 served as an endogenous control for miR7111-5p and miR6870-5p and GAPDH used as a reference control for $S R F$ target gene. The primer sequences are summarized in Table 2. The formula $2^{-\Delta \mathrm{Cq}}$ was utilized to determine the expression level of miR7111-5p, miR6870-5p, and SRF.[18] 


\begin{tabular}{|c|c|c|c|}
\hline \multicolumn{2}{|c|}{ Clinicopathological parameter } & Total cases $(n=30)$ & $\mathbf{p}$ \\
\hline \multicolumn{4}{|c|}{ Age, years } \\
\hline$>40$ & & 27 & 0.890 \\
\hline$<40$ & & 2 & \\
\hline \multicolumn{4}{|l|}{ Gender } \\
\hline Male & & 23 & 0.318 \\
\hline Fema & & 7 & \\
\hline \multicolumn{4}{|c|}{ Smoke } \\
\hline Non- & hokers & 22 & 0.555 \\
\hline Smok & & 7 & \\
\hline Ex-sn & kers & 1 & \\
\hline \multicolumn{4}{|c|}{ Clinical stage } \\
\hline 1 & & 3 & 0.013 \\
\hline ॥ & & 3 & \\
\hline III & & 7 & \\
\hline IV & & 17 & \\
\hline \multicolumn{4}{|c|}{ Pathological grading } \\
\hline 1 & & 17 & 0.333 \\
\hline ॥ & & 13 & \\
\hline \multicolumn{4}{|c|}{ Tumor size $(\mathrm{cm})$} \\
\hline$<2$ & & 3 & 0.693 \\
\hline $2-5$ & & 19 & \\
\hline$>5$ & & 8 & \\
\hline \multicolumn{4}{|c|}{ Vascular invasion } \\
\hline Yes & & 6 & 0.325 \\
\hline No & & 24 & \\
\hline \multicolumn{4}{|c|}{ Necrosis presence } \\
\hline Yes & & 7 & 0.777 \\
\hline No & & 23 & \\
\hline \multicolumn{4}{|c|}{ Family history } \\
\hline Yes & & 6 & 0.045 \\
\hline No & & 24 & \\
\hline \multicolumn{4}{|c|}{ Metastasis } \\
\hline Positi & & 19 & 0.880 \\
\hline Nega & & 11 & \\
\hline \multicolumn{4}{|c|}{ Lymph node metastasis } \\
\hline Yes & & 5 & 0.886 \\
\hline No & & 24 & \\
\hline Unkn & & 1 & \\
\hline \multicolumn{4}{|c|}{ Perineual invasion } \\
\hline Yes & & 11 & 0.403 \\
\hline No & & 19 & \\
\hline
\end{tabular}

SRF: Serum response factor; OSCC: Oral squamous cell carcinoma

\section{Statistical Analysis}

All experiments were repeated three times, and numerical data are expressed as the means \pm standard deviation. Statistical analysis was performed using GraphPad Prism software 5.0 (GraphPad Software, Inc., San Diego, CA, USA) and SPSS (GraphPad Prism 5 software (version 18.0; SPSS, Inc., Chicago,
Table 2 Sequence of the primers used in this study

\begin{tabular}{|c|c|c|}
\hline Primers & Primer sequence & $\begin{array}{c}\text { Accession } \\
\text { number }\end{array}$ \\
\hline MiR7111 & F:UGGGGGAGGAAGGACAGGCCAUA & 4487303 \\
\hline$-5 p$ & R: GTGCAGGGTCCGAGGT* & \\
\hline MiR6870 & F: UGGGGGAGAUGGGGGUUGA & 4487303 \\
\hline$-5 p$ & R: GTGCAGGGTCCGAGGT* & \\
\hline \multirow[t]{2}{*}{ SRF } & F: ACTGCCTTCAGTAGGAACAA & 4487303 \\
\hline & R: CGACCTTCAGCAAGAGGAAGACGGGC & \\
\hline \multirow[t]{2}{*}{ U6 } & F: CTCGCTTCGGCAGCACA & 4487303 \\
\hline & R: AACGCTTCACGAATTTGCGT & \\
\hline \multirow[t]{2}{*}{ GAPDH } & F: GTCTCCTCTGACTTCAACAGCG & 4487303 \\
\hline & R: ACCACCCTGTTGCTGTAGCCAA & \\
\hline
\end{tabular}

*The reverse primer sequence was taken from Chen et al.,[19]; SRF: Serum response factor

IL, USA). Expression data were controlled for normal distribution by one-sample Kolmogorov-Smirnov (K-S test). A one-way ANOVA was used to determine statistically differences in SRF gene expression levels in different tumor sizes. The associations between miR7111-5p, miR6870-5p, and SRF levels and clinic-pathological parameters of OSCC patients were assessed using independent sample test and independent sample Kruskal-Wallis test. The correlation between miR7111-5p, miR6870-5p, and SRF expression was performed by Pearson correlation analysis. $\mathrm{p}<0.05(\leq 0.05)$ was considered to indicate a statistically significant.

\section{Results}

MiR7111-5p and miR6870-5p Expression Analysis by RT-qPCR

To further study the association between miR7111-5p and miR6870-5p expression signature in OSCC, miRs expression levels were detected in 30 paired OSCC tissues and adjacent normal oral epithelial tissues using RT-qPCR. In this study, both miR7111-5p and miR6870-5p expressions were markedly downregulated in OSCC tissues compared with in normal tissues $(\mathrm{p}<0.01)$ (Figs. 1a, b).

\section{SRF Expression Analysis by RT-qPCR}

In the present study, the potential targets of miR7111$5 p$ and miR6870-5p were identified with promising miRNA-target prediction tools (The TargetScan, Mirwalk, and Mirdb). According to conserved seed matching sequence principles, the predicted miRs and potential target gene were selected by identi- 


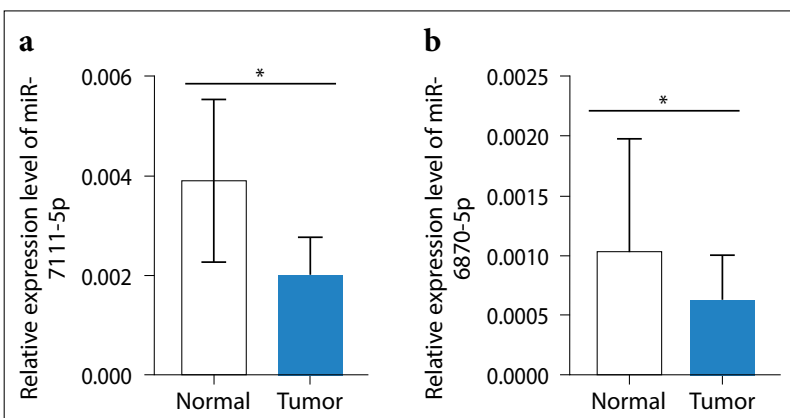

Fig. 1. Downregulated expressions of miR-7111-5p (a) and miR-6870-5p (b) in oral squamous cell carcinoma. Data are shown as means \pm SD of three separate experiments. ${ }^{*} \mathrm{p}<0.05$.

fying the intersection of databases.[20] $S R F$ was identified as a potential target for miR7111-5p and miR6870-5p based on predicted databases. We used qRT-PCR to investigate SRF expression in 30 pairs of OSCC tissues and adjacent normal oral epithelial tissues. Statistical analysis revealed that $S R F$ expression level was significantly upregulated ( $\sim 5$-folds) in OSCC tissues compared with that in adjacent normal tissues ( $\mathrm{p}=0.025$ ) (Fig. 2). Furthermore, the expression level of $S R F$ in OSCC tissues was negatively correlated with corresponding tumor-adjacent normal tissues $(\mathrm{p}=0.241)$.

\section{The Association between SRF Expression and Clini- copathological Characteristics}

To examine the potential diagnostic value of $S R F$ expression rate in OSCC, we analyzed the SRF expression levels at OSCC patients with different clinical feature categories (Table 1). The results revealed that $S R F$ expression was significantly associated with clinical tumor stages $(\mathrm{p}=0.020)$ and family history $(\mathrm{p}=0.045)$. The patients were assessed for the history of cancer in the family in which $20 \%(n=6)$ of the patients had a family history of cancer, and $80 \%(n=24)$ with no familial history. The expression of SRF was increased in all patients and was higher in patients with a family history of cancer. A significant association was found between SRF upregulation and family history of cancer $(\mathrm{p}<0.05)$. We found high expression of $S R F$ was not statistically associated with clinical features including lymph node status $(\mathrm{p}=0.88)$, tumor grade and size $(\mathrm{p}=0.33, \mathrm{p}=0.69)$, vascular invasion $(\mathrm{p}=0.32)$, necrosis $(\mathrm{p}=0.77)$, metastasis $(p=0.88)$, perineural invasion $(\mathrm{p}=0.40)$, sex $(\mathrm{p}=0.31)$, and smoking $(\mathrm{p}=0.55)$.

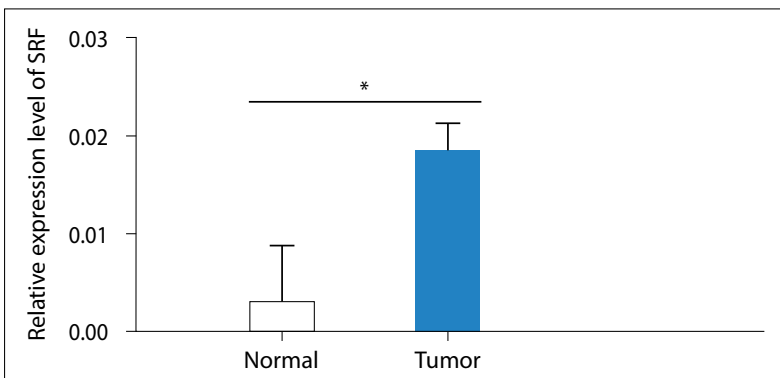

Fig. 2. Expression level of serum response factor showed upregulation in oral squamous cell carcinoma $(\mathrm{p}=0.025)$. Data are shown as means \pm SD of three separate experiments. ${ }^{*} \mathrm{p}<0.05$.

SRF: Serum response factor.

The Correlation between the Expression of miR71115p, miR6870-5p and SRF

To study miR7111-5p, miR6870-5p levels positively and negatively correlated to $S R F$ expression in OSCC, a correlation analysis by Pearson method performed in 30 pairs OSCC tissues and adjacent normal tissues. We did not find a significant correlation between downregulation of miR7111-5p, miR6870-5p, and increased expression of $S R F$ gene in OSCC tissues and adjacent normal tissues (Fig. 3a, b).

\section{Discussion}

It is widely recognized that the dysregulation of miRNAs through different cellular pathways contributes to malignant transformation and tumor progression of different cancer entities.[21] Accumulating evidence indicates that aberrant expression of miRNAs, either overexpression or underexpression, leads to the initiation and progression of OSCC.[22]

Many miRNAs, including miR-10b,[23] miR21,[24] miR-31,[25] miR-34b/c,[26] miR-146a-5p,[15] miR186,[21] miR-196a/b,[16] miR-223, miR-320, miR-494,[21] miR-543,[27] miR-1179[14], and miR4513 ,[28] have been reported to be dysregulated in OSCC. miR7111-5p and miR6870-5p have been confirmed to be upregulated in hepatocellular carcinoma through targeting the KSR2 gene.

To date, no study has attempted to evaluate the expression of MiR7111-5p and miR6870-5p in OSCC, except the research by Chamorro-Petronacci et al.,[8] in which only microarray-based miRNA expression profiling was taken into account and found to be upregulated. In the present study, qRT-PCR was employed to evaluate dysregulated expression levels of miR7111-5p, miR6870-5p in OSCC. 


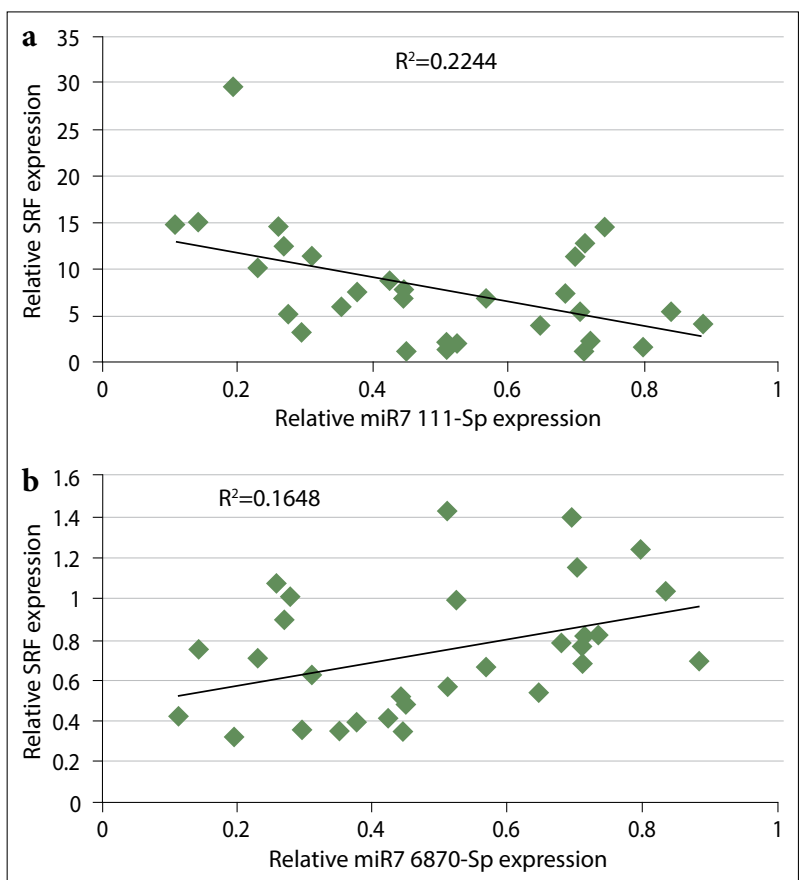

Fig. 3. No significant correlation was found between the expression of serum response factor, miR-7111$5 \mathrm{p}$ (a) and miR-7111-5p (b) in oral squamous cell carcinoma patients' tissues. ${ }^{*} \mathrm{p}<0.05$. SRF: Serum response factor.

Our results showed that miR7111-5p, miR6870-5p downregulated in OSCC patients which were not in a manner consistent with the upregulated level obtained from the previous report. miRNAs perform their regulatory roles through the translation repression or the degradation of their target mRNAs. Therefore, recognition of regulation relations of miRNAs on target genes for a specific type of cancer can be a crucial step in early detection and to improve patient survival rates.

Regardless of the regulatory function of $S R F$ at the expression of various miRNAs involved in the MAPKinase Signaling Pathway, it has been demonstrated to be a direct target for regulation by many miRNAs in several types of cancer.[15] $S R F$ was selected as a potential target gene for miR7111-5p and miR6870$5 \mathrm{p}$ based on analysis using TargetScan, Mirwalk and, miRDB online bioinformat-ics software.

Although research of the deregulated functions of miR7111-5p and miR6870-5p in different cancers is in progress, their regulatory function by targeting $S R F$ in OSCC still remains largely unclear. To the best of our knowledge, this is the first evaluation of the correlation between deregulated expression levels of miR7111-5p, miR6870-5p, and SRF in OSCC. We also investigated the correlation between clinico-pathological stages of OSCC and SRF expression.

Our results revealed that the miR7111-5p and miR6870-5p expression levels were significantly decreased in OSCC compared with adjacent normal tissues. Moreover, SRF expression was significantly upregulated in OSCC and was not correlated with downregulated expression levels of miR7111-5p and miR6870-5p.

Family history has been reported as an important risk factor for oral cavity cancer and many of cancers. Therefore, understanding the relationship between family history and oral cavity cancer provides great potential in screening and preventive strategies resulting reduce the risk of cancer.[29,30] In our study among investigated clinicopathological characteristics, the upregulation of $S R F$ only was significantly correlated with tumor stage $(\mathrm{p}=0.020)$ and family history $(\mathrm{p}=0.045)$ probably due to the small sample size and needs to further research in the future.

According to the results of the present study, the expression of SRF gene has increased in higher stages (IV) which may be a potential marker for early detection of OSCC in the future. OSCC is among the most common causes of cancer death in the world, accounting for more than $90 \%$ of all oral cancers and has a 5 -year survival rate of $<50 \%$.

Ghafari et al.[31] revealed that a changing trend of histopathologic pattern with an increasing number of moderately differentiated OSCC and a higher increasing rate in tumor size and vascular, perineural invasion during years of 2006-2015 in Iran. Altered expression miRNAs have been implicated in various forms of cancer. Therefore, identification of miRNAs as a biomarker may provide new insights into clinical diagnosis at earlier stages and treatment of OSCC. $[32,33]$ The identification of OSCC patients in the advanced clinical stage provides the main obstacle to correct treatment and save lives in Iran. Therefore, our findings can be an effective step in creating specific miRNAs signatures in predicting and diagnosing of OSCC.

Our study suggested that downregulation of miR7111-5p and miR-6870-5p alone and or with other altered expression miRNAs profiles may serve as a prognostic marker to predict and early detection of OSCC. Given that, the relatively small sample size was a major limitation in this study, therefore whether miRs down expression impacted the expression of $S R F$ and or $S R F$ acts as oncogene contributes to OSCC requires further investigation. 


\section{Conclusion}

In the present study, the expression of $S R F$ target gene as a possible biomarker involved in OSCC was quantitatively examined in OSCC tissues compared with adjacent normal tissues and obtained result was consistent with $S R F$ expression changes in other investigated cancers. Downregulated expression levels of miR-7111-5p and miR-6870-5p as well as SRF overexpression in OSCC patients identified in the present study may serve as biomarkers for the detection and monitoring of OSCC. Further studies with large samples could reflect and confirm more clarifying results.

Peer-review: Externally peer-reviewed.

Conflict of Interest: All authors declared no conflict of interest.

Ethics Committee Approval: The study was approved by the Research Medical Ethics Committee of Imam Khomeini Hospital (No: IR.IAU.SRB.REC.1399.099, Date: 19/11/2020).

Financial Support: None declared.

Authorship contributions: Concept - F.J.; Design - F.J.; Supervision - F.J.; Funding - M.S.; Materials - M.S.; Data collection and/or processing - M.S.; Data analysis and/or interpretation - M.S.; Literature search - A.G.; Writing - A.G.; Critical review - A.G.

\section{References}

1. Gharat SA, Momin M, Bhavsar C. Oral squamous cell carcinoma: Current treatment strategies and nanotechnology-based approaches for prevention and therapy. Crit Rev Ther Drug Carrier Syst 2016;33(4):363-400.

2. Malik UU, Zarina S, Pennington SR. Oral squamous cell carcinoma: Key clinical questions, biomarker discovery, and the role of proteomics. Arch Oral Biol 2016;63:53-65.

3. Zygogianni AG, Kyrgias G, Karakitsos P, Psyrri A, Kouvaris J, Kelekis N, et al. Oral squamous cell cancer: Early detection and the role of alcohol and smoking. Head Neck Oncol 2011;3:2.

4. Alberto PL, Kessler HP. Early diagnosis and treatment of squamous cell carcinoma-a case comparison highlighting its importance. Biol Med (Aligarh) 2016;8:273.

5. Henry NL, Hayes DF. Cancer biomarkers. Mol Oncol 2012;6(2):140-6.

6. Hui Y, Li Y, Jing Y, Feng JQ, Ding Y. miRNA-101 acts as a tumor suppressor in oral squamous cell carcinoma by targeting CX chemokine receptor 7. Am J Transl Res 2016;8(11):4902-11.

7. $\mathrm{Wu} \mathrm{BH}$, Xiong XP, Jia J, Zhang WF. MicroRNAs:
New actors in the oral cancer scene. Oral Oncol 2011;47(5):314-9.

8. Chamorro-Petronacci C, Pérez-Sayáns M, PadinIruegas ME, Suarez-Peñaranda JM, Lorenzo-Pouso $\mathrm{AI}$, Blanco-Carrion $\mathrm{A}$, et al. Association of miR497-5p and miR-4417 expression in oral squamous cell carcinoma and clinical parameters. Preprints 2018;2018:100331.

9. Yang CC, Hung PS, Wang PW, Liu CJ, Chu TH, Cheng HW, et al. miR-181 as a putative biomarker for lymph-node metastasis of oral squamous cell carcinoma. J Oral Pathol Med 2011;40(5):397-404.

10. Hung PS, Tu HF, Kao SY, Yang CC, Liu CJ, Huang TY, et al. miR-31 is upregulated in oral premalignant epithelium and contributes to the immortalization of normal oral keratinocytes. Carcinogenesis 2014;35(5):1162-71.

11. Hu J, Xu JF, Ge WL. miR-497 enhances metastasis of oral squamous cell carcinoma through SMAD7 suppression. Am J Transl Res 2016;8(7):3023-31.

12. Ghffari M, Asadi M, Shanaehbandi D, Bornehdeli S, Sadeghzadeh M, Khani HM, et al. Aberrant expression of miR-103, miR-184, miR-378, miR-497 and miR-506 in tumor tissue from patients with oral squamous cell carcinoma regulates the clinical picture of the patients. Asian Pac J Cancer Prev 2020;21(5):1311-5.

13. Fei Y, Shan W, Chen X. miR-503-5p functions as an oncogene in oral squamous cell carcinoma by targeting smad7. Histol Histopathol 2020;35(8):893-901.

14. Min A, Zhu C, Peng S, Rajthala S, Costea DE, Sapkota D. MicroRNAs as important players and biomarkers in oral carcinogenesis. Biomed Res Int 2015;2015:186904.

15.Zhang X, Azhar G, Helms SA, Wei JY. Regulation of cardiac microRNAs by serum response factor. J Biomed Sci 2011;18(1):15.

16. Liu N, Bezprozvannaya S, Williams AH, Qi X, Richardson JA, Bassel-Duby R, et al. microRNA-133a regulates cardiomyocyte proliferation and suppresses smooth muscle gene expression in the heart. Genes Dev 2008;22:3242-54.

17. Buller B, Chopp M, Ueno Y, Zhang L, Zhang RL, Morris $\mathrm{D}$, et al. Regulation of serum response factor by miRNA-200 and miRNA-9 modulates oligodendrocyte progenitor cell differentiation. Glia 2012;60(12):190614.

18. Chi XJ, Wei LL, Bu Q, Mo N, Chen XY, Lan D, et al. Identification of high expression profiles of miR-31-5p and its vital role in lung squamous cell carcinoma: A survey based on qRT-PCR and bioinformatics analysis. Transl Cancer Res 2019;8:788-801.

19. Chen C, Ridzon DA, Broomer AJ, Zhou Z, Lee DH, Nguyen JT, et al. Realtime quantification of $\mathrm{mi}$ croRNAs by stem-loop RT-PCR. Nucleic Acids Res 2005;33:e179. 
20. Bowen L, Feng W, Xiang L, Shuai S, Yuehong S, Hongyu Y. Hsa_circ_0008309 may be a potential biomarker for oral squamous cell carcinoma. Dis Markers 2018;2018:7496890.

21. Ries J, Vairaktaris E, Agaimy A, Kintopp R, Baran C, Neukam FW, et al. miR-186, miR-3651 and miR494: Potential biomarkers for oral squamous cell carcinoma extracted from whole blood. Oncol Rep 2014;31(3):1429-36.

22. Jin Y, Li Y, Wang X, Yang Y. Dysregulation of MiR519d affects oral squamous cell carcinoma invasion and metastasis by targeting MMP3. J Cancer 2019;10(12):2720-34.

23. Severino P, Brüggemann $\mathrm{H}$, Andreghetto FM, Camps C, de Fatima Garrido Klingbeil M, de Pereira WO, et al. MicroRNA expression profile in head and neck cancer: HOX-cluster embedded microRNA-196a and microRNA-10b dysregulation implicated in cell proliferation. BMC Cancer 2013;13:533.

24. Singh P, Srivastava AN, Sharma R, Mateen S, Shukla B, Singh A, et al. Circulating MicroRNA-21 expression as a novel serum biomarker for oral sub-mucous fibrosis and oral squamous cell carcinoma. Asian Pac J Cancer Prev 2018;19(4):1053-7.

25. Liu CJ, Lin SC, Yang CC, Cheng HW, Chang KW. Exploiting salivary miR-31 as a clinical biomarker of oral squamous cell carcinoma. Head Neck 2012;34(2):21924.

26. Ren, Z, Wu, K, Yang, R. Liu ZQ, Cao W. Differential expression of matrix metalloproteinases and miRNAs in the metastasis of oral squamous cell carcinoma.
BMC Oral Health 2020;20(1):24.

27. Wang L, Chen W, Zha J, Yan Y, Wei Y, Chen X, et al. miR-543 acts as a novel oncogene in oral squamous cell carcinoma by targeting CYP3A5. Oncol Rep 2019;42(3):973-90.

28. Xu YX, Sun J, Xiao WL, Liu YS, Yue J, Xue LF, et al. MiR4513 mediates the proliferation and apoptosis of oral squamous cell carcinoma cells via targeting CXCL17. Eur Rev Med Pharmacol Sci 2019;23(9):3821-8.

29. Radoï L, Paget-Bailly S, Guida F, Cyr D, Menvielle G, Schmaus A, et al. Family history of cancer, personal history of medical conditions and risk of oral cavity cancer in France: The ICARE study. BMC Cancer 2013;13:560.

30. Acheson LS, Wang C, Zyzanski SJ, Lynn A, Ruffin MT, Gramling R, et al. Family history and perceptions about risk and prevention for chronic diseases in primary care: A report from the family healthware impact trial. Genet Med 2010;12(4):212-8.

31. Ghafari R, Naderi NJ, Razavi AE. A retrospective institutional study of histopathologic pattern of oral squamous cell carcinoma (OSCC) in Tehran, Iran during 2006-2015. J Res Med Sci 2019;24:53.

32. Wu Q, Cao R, Chen J, Xie X. Screening and identification of biomarkers associated with clinicopathological parameters and prognosis in oral squamous cell carcinoma. Exp Ther Med 2019;18(5):3579-87.

33. Li L, Cheng Y, Lin L, Liu Z, Du S, Ma L, et al. Global analysis of miRNA signature differentially expressed in insulin-resistant human hepatocellular carcinoma cell line. Int J Med Sci 2020;17(5):664-77. 